\title{
Prognostic scores and biomarkers for pediatric community-acquired pneumonia: how far have
} we come?

\author{
Samuel N Uwaezuoke' \\ Adaeze C Ayuk ${ }^{2}$ \\ 'Pediatric Nephrology Firm, ${ }^{2}$ Pediatric \\ Pulmonology Firm, Department of \\ Pediatrics, University of Nigeria \\ Teaching Hospital, Ituku-Ozalla, \\ Enugu, Nigeria
}

\section{Video abstract}

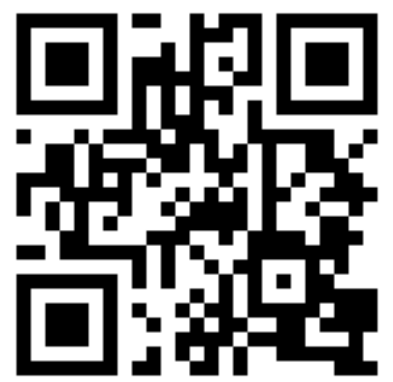

Point your Smart Phone at the code above. If you have a QR code reader the video abstract will appear. Or use: http://youtu.be/TD08]B616xw
Correspondence: Samuel N Uwaezuoke Pediatric Nephrology Firm, Department of Pediatrics, University of Nigeria Teaching Hospital, Ituku-Ozalla, PMB 0I I29, Enugu 40000I, Nigeria Tel +2348033248108

Email snuwaezuoke@yahoo.com
This article was published in the following Dove Press journal:

Pediatric Health, Medicine and Therapeutics

20 February 2017

Number of times this article has been viewed

\begin{abstract}
This article aimed to review the current prognostic and diagnostic tools used for community-acquired pneumonia (CAP) and highlight those potentially applicable in children with CAP. Several scoring systems have been developed to predict CAP mortality risk and serve as guides for admission into the intensive care unit. Over the years, clinicians have adopted these tools for improving site-of-care decisions because of high mortality rates in the extremes of age. The major scoring systems designed for geriatric patients include the Pneumonia Severity Index and the confusion, uremia, respiratory rate, blood pressure, age $>65$ years (CURB-65) rule, as well as better predictors of intensive care unit admission, such as the systolic blood pressure, multilobar chest radiography involvement, albumin level, respiratory rate, tachycardia, confusion, oxygenation and arterial pH (SMART-COP) score, the Infectious Diseases Society of America/American Thoracic Society guidelines, the criteria developed by España et al as well as the systolic blood pressure, oxygenation, age and respiratory rate (SOAR) criteria. Only the modified predisposition, insult, response and organ dysfunction (PIRO) score has so far been applied to children with CAP. Because none of the tools is without its limitations, there has been a paradigm shift to incorporate biomarkers because they are reliable diagnostic tools and good predictors of disease severity and outcome, irrespective of age group. Despite the initial preponderance of reports on their utility in geriatric CAP, much progress has now been made in demonstrating their usefulness in pediatric CAP.
\end{abstract}

Keywords: community-acquired pneumonia, children, scoring systems, biomarkers

\section{Introduction}

Community-acquired pneumonia (CAP) refers to pneumonia in a previously healthy patient who acquired the infection in the community, as differentiated from hospitalacquired pneumonia (HAP). ${ }^{1}$ Ewig however believes CAP does not describe pneumonia acquired in the community per se but represents a concept about a distinct clinical syndrome in distinct community patients. ${ }^{2}$

It is one of the most common serious infections in childhood, with an estimated incidence of 34-40 cases per 1,000 children in Europe and North America. ${ }^{3-5}$ Although CAP-related mortality is relatively rare in developed countries, the picture is different in developing countries where pneumonia is one of the major causes of childhood mortality. ${ }^{6}$ Nevertheless, severe CAP remains the single main cause of mortality from infectious diseases in developed countries.

With respect to etiology, the basic microbial patterns typically consist of Streptococcus pneumoniae, an atypical group, a non-pneumococcal non-atypical group 
and the Gram-negative group. ${ }^{2}$ The relative frequency of each group is determined by the severity of pneumonia, age, comorbidity, seasonality and individual risk factors. ${ }^{2}$ For instance, in developing countries, a causal relationship exists between severe and life-threatening pneumonias and bacterial agents such as S. pneumoniae, Haemophilus influenzae and Staphylococcus aureus. ${ }^{2}$ A similar trend also occurs in Europe and North America. ${ }^{7}$ Secondly, agespecific etiologic patterns have been established; Group B streptococcus and Gram-negative enteric bacteria are the most common pathogens in the neonatal age group. Beyond this age bracket, $S$. pneumoniae remains the most common bacterial agent, while Mycoplasma pneumoniae and Chlamydia pneumoniae (atypical group) are frequently associated with illness in preschool-aged children and are common causative bacteria in older children and adolescents. ${ }^{8,9}$ In European countries such as Germany, Legionella spp. (especially L. pneumonia and L. pneumophila) have been reported as prominent etiologic agents, especially in adult patients with CAP. ${ }^{10,11}$ Elsewhere in China, the bacteria have also been noted in the list of common causative pathogens according to a multicenter study, ${ }^{12}$ while a recent systematic review revealed their contributory role in causing CAP in Asia and the Middle East. ${ }^{13}$ Despite the need for hospitalization in only a minority of patients, several severity-of-illness tools have been developed, which can help in deciding on admission, especially in the intensive care units (ICUs). In contrast to what is obtainable in geriatric CAP, the absence of a pediatric CAP severity score remains one of the unresolved issues about the disease. In fact, little progress has been made in establishing CAP-specific scores in children. Over the years, clinicians have adopted prognostic scoring systems for improving site-of-care decisions because of high mortality rates in the extremes of age. Precise risk stratification of patients, used currently, aimed at ensuring appropriate site-of-care decisions has its shortcomings. Therefore, the procedure has been improved by the inclusion of several biomarkers in order to differentiate viral from bacterial infections, restrict the duration of antibiotic therapy and function as prognostic indicators. ${ }^{14}$ With technological advancement, these biomarkers hold great prospects for aiding critical decisions for patients with pneumonia. ${ }^{15}$ Moreover, the combination of several biomarkers reflecting different pathophysiological pathways can potentially improve the management of CAP in future. ${ }^{16}$ Interestingly, the prognostic and diagnostic utility of these biomarkers has been demonstrated in both geriatric and pediatric CAP.
This article aims to review these prognostic and diagnostic tools, in addition to highlighting those potentially applicable in children with CAP.

\section{Risk stratification of patients with CAP: the current scoring systems}

The main determinants of prognosis in patients with CAP are respiratory failure, sepsis-associated organ dysfunction and unstable comorbidities. Thus, the current risk stratification tools have been fundamentally designed to predict mortality and identify low-risk patients who are potentially suitable for outpatient management. ${ }^{17}$

This underscores the importance of site-of-care decisions in the management of these patients. Several scoring systems have been developed to predict mortality risk in CAP, and these have been used as guides for patient hospitalization or admission in the ICU. ${ }^{18}$ The most extensively evaluated scoring systems are the Pneumonia Severity Index (PSI) and the confusion, uremia, respiratory rate, BP, age $>65$ years (CURB-65) rule (a modification of the British Thoracic Society [BTS] rule), both of which are basically appropriate in adult patients.

\section{Pneumonia Severity Index}

The PSI was introduced to assist in identifying patients with CAP who may either require ambulatory (outpatient) or hospitalized (inpatient) treatment. ${ }^{19}$ This prognostic tool is based on 20 clinical variables that group the patients into five risk categories (Risk Classes I-V) and could be utilized to predict 30-day survival. Although the PSI performs well as a predictor of mortality, it is time consuming and more complex as it requires multiple clinical and laboratory parameters. Furthermore, the PSI was developed to identify low-mortality-risk patients and may underestimate the severity of CAP. ${ }^{20}$ For instance, a Risk Class I or II pneumonia patient may require outpatient treatment. A Risk Class III patient (after evaluating other factors such as home environment and follow-up) may need either outpatient or inpatient treatment and surveillance for a short duration, while Risk Class IV and V patients should be hospitalized for treatment (Figure 1).

Since PSI may underestimate the severity of illness (which is a limitation), the simpler CURB-65 rule was developed to identify more severely ill patients with ease.

\section{CURB-65 and CRB-65 scoring systems}

The CURB-65 parameters consist of the following items from which the acronym was derived: new-onset mental confusion, urea $>7 \mathrm{mmol} / \mathrm{L}$, respiratory rate $\geq 30$ breaths $/ \mathrm{min}$, 


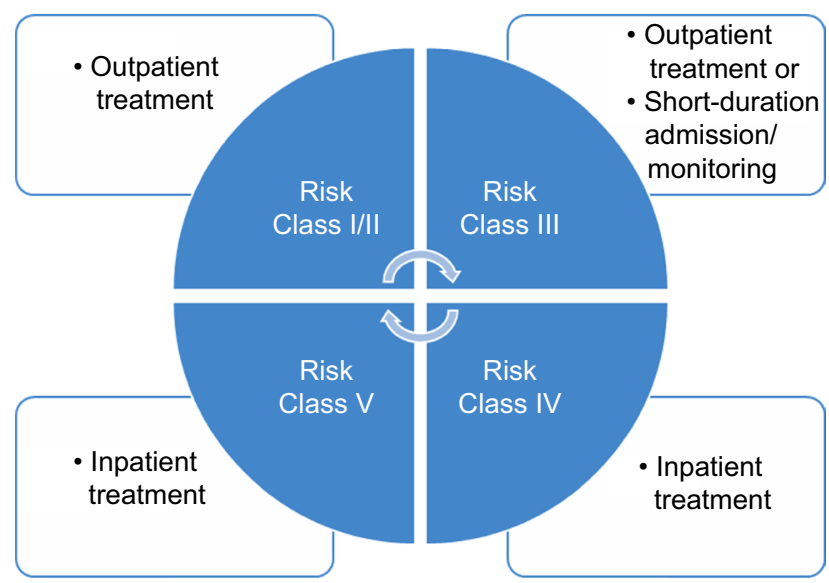

Figure I Risk stratification of patients with community-acquired pneumonia and treatment algorithm using Pneumonia Severity Index scoring system.

systolic blood pressure $<90 \mathrm{mmHg}$ or diastolic blood pressure $\leq 60 \mathrm{mmHg}$ and age $\geq 65$ years. $^{21}$ It is a 5-point score with three risk categories: low risk ( $0-1$ points), intermediate risk ( 2 points) and high risk ( $>3$ points). Unlike PSI, this tool is more straightforward and also constitutes an accurate tool for the prediction of 30-day survival. ${ }^{18}$ The CURB-65 can be further simplified by the omission of the laboratory parameter blood urea nitrogen (BUN), giving rise to only the CRB-65 criteria.

A comparison of the CURB-65 and CRB-65 scoring systems has been made in two studies. ${ }^{22,23}$ One of the studies reported that both the CURB-65 and the CRB-65 were equally effective in predicting 30 -day mortality, ${ }^{22}$ while in the other study, the investigators noted that both CRB-65 and CURB-65 could predict 30-day survival, even though the CRB-65 tended to underestimate mortality risk: defining $26 \%$ of the patients who died as low risk. ${ }^{23}$ Therefore, it was concluded that the more convenient and readily available CRB-65 should be used in ambulatory patients, while the full CURB-65 rule should be applied to hospitalized patients. ${ }^{23}$

\section{PSI versus CURB-65}

Interestingly, further comparisons between the PSI and the CURB-65 tools have brought to the fore important differences in terms of advantages and limitations. The development and validation of PSI were based on the need to identify low-mortality-risk patients. Thus, this tool can potentially be misleading for site-of-care decisions and can underestimate severity of illness, more especially in young patients who do not have comorbid illnesses. ${ }^{19,24}$ Conversely, the CURB-65 scoring system may be optimal for identifying high-mortality-risk patients with severe CAP who otherwise might be missed without formal assessment of barely noticeable deviations in key vital signs. ${ }^{21,24}$ Hence, both scoring systems assess pneumonia and mortality risk from different perspectives, and each is best suited for identifying patients at opposite ends of the CAP-severity spectrum. ${ }^{18}$

A review of some studies that have compared the PSI and CURB-65 tools in the same population shows that in one report, ${ }^{25}$ both tools were good at predicting mortality and in identifying low-risk patients. However, the CURB-65 appeared to be more distinctive in defining mortality risk in severe CAP. ${ }^{25}$ In another study, both the PSI and the CURB-65 were used to evaluate a large number of hospitalized and ambulatory patients with CAP. ${ }^{22}$ The CURB-65 (and the simpler CRB-65 version) accurately predicted 30-day mortality, the need for mechanical ventilation and perhaps the need for hospitalization. ${ }^{22}$ Furthermore, the CURB-65 criteria correlated with the time to clinical stability. While the PSI predicted mortality, it was not good at predicting the need for ICU admission. The authors also documented that the CURB-65 tool could not predict the need for ICU admission, although it was observed to be more accurate than the PSI for this site-of-care decision. ${ }^{22}$

It is therefore important to consider other tools that have more predictive value when deciding the need for ICU admission.

\section{Infectious Diseases Society of America (IDSA)/American Thoracic Society (ATS) criteria}

Some researchers ${ }^{26}$ evaluated the ATS guidelines, ${ }^{27}$ which proposed 10 criteria to define severe CAP. It was established that the requirement for ICU was defined by the presence of two of three minor criteria (systolic blood pressure $\leq 90 \mathrm{mmHg}$, multilobar disease or the ratio of partial pressure of arterial oxygen to fractional inspired oxygen $\left[\mathrm{PaO}_{2} /\right.$ $\mathrm{FiO}_{2}$ ratio] $\leq 250$ ) or one of two major criteria (need for mechanical ventilation or septic shock). ${ }^{26}$ Obviously, this rule had better sensitivity and specificity for defining the requirement for ICU admission than either the PSI or the BTS rule in view of the estimated sensitivity of $69 \%$ and specificity of $98 \%$. Based on these findings, the 2001 ATS guidelines for CAP subsequently recommended that severe CAP could be defined on the basis of the presence of these major and minor criteria. ${ }^{27}$ Against this backdrop, the IDSA/ ATS committee released guidelines in 2007, which expanded the criteria for ICU admission to include the presence of at least three of the following nine minor criteria: $\mathrm{PaO}_{2} / \mathrm{FiO}_{2}$ ratio $<250$, respiratory rate $>30$ breaths $/ \mathrm{min}$, confusion, multilobar infiltrates, systolic blood pressure $<90 \mathrm{mmHg}$ despite aggressive fluid resuscitation, BUN $>20 \mathrm{mg} / \mathrm{dL}$, leukopenia 
$\left(<4,000\right.$ cells $\left./ \mathrm{mm}^{3}\right)$, thrombocytopenia $\left(<100,000\right.$ cells $\left./ \mathrm{mm}^{3}\right)$ and hypothermia $\left(<36^{\circ} \mathrm{C}\right) .{ }^{28}$ This scoring system nevertheless requires further validation.

\section{Criteria developed by España et al}

Another risk stratification tool is the severe CAP model proposed by España et al. ${ }^{29}$ Having examined records from 1,057 patients, the investigators established that the need for admission into the ICU was defined by the presence of one of two major criteria: arterial $\mathrm{pH}<7.30$ or systolic blood pressure $<90 \mathrm{mmHg} .{ }^{29} \mathrm{In}$ the absence of these criteria, severe CAP could also be identified by the presence of two of six of the following minor criteria: confusion, BUN $>30 \mathrm{mg} /$ $\mathrm{dL}$, respiratory rate $>30$ breaths $/ \mathrm{min}, \mathrm{PaO}_{2} / \mathrm{FiO}_{2}$ ratio $<250$, multilobar infiltrates and age of at least 80 years. When these criteria were fulfilled, the scoring system was $92 \%$ sensitive for identifying those with severe disease and was more accurate than the PSI or CURB-65 criteria, although not quite as specific as the CURB-65 rule. ${ }^{29}$

\section{SMART-COP scoring system}

The systolic blood pressure, multilobar chest radiography involvement, albumin level, respiratory rate, tachycardia, confusion, oxygenation and arterial pH (SMART-COP) scoring system is another current tool for predicting the need for ICU admission. It was a product of the Australian CAP study that defined the need for intensive respiratory or vasopressor support (IRVS), which was seen as a more objective end point than admission into the ICU. ${ }^{30}$ The SMART-COP rule (the acronym for systolic blood pressure $<90 \mathrm{mmHg}$, multilobar infiltrates, albumin $<3.5 \mathrm{~g} / \mathrm{dL}$, respiratory rate elevation $[\geq 25$ breaths $/ \mathrm{min}$ for those age $\leq 50$ years, and $\geq 30$ breaths $/ \mathrm{min}$ for those age $>50$ years], Tachycardia $[>125$ beats $/ \mathrm{min}]$, confusion, low oxygen $[<70 \mathrm{mmHg}$ if age $\leq 50$ years or $<60 \mathrm{mmHg}$ if age $>50$ years] and arterial $\mathrm{pH}<7.35$ ) performed well for patients under IRVS who were initially admitted into the ICU, as well as for those who were transferred to the ICU after initial admission in the ward. Notably, one study has demonstrated that SMART-COP was superior to the other prognostic scoring tools for predicting the need for IRVS, with a sensitivity of $85 \%$ compared to $55 \%$ for CURB-65 $\geq 3$ or a PSI class $\geq \mathrm{IV}^{31}$

\section{Systolic blood pressure, oxygenation, age and respiratory rate (SOAR) criteria}

Finally, a specific scoring system for the elderly with the acronym SOAR has also been developed. ${ }^{32}$ The best predictor of severe CAP and mortality was the presence of two of four
SOAR criteria: systolic blood pressure $<90 \mathrm{mmHg}, \mathrm{PaO}_{2} / \mathrm{FiO}_{2}$ $<250$, age $>65$ years and respiratory rate $>30$ breaths $/ \mathrm{min}$. When two criteria were present, the sensitivity for predicting mortality from CAP was $81 \%$, and the specificity was $60 \%{ }^{32}$ This tool was developed in place of CURB- 65 because many geriatric patients have an elevated BUN and confusion, and thus the discriminating value of these parameters is limited in older subjects. ${ }^{17}$

\section{Modified predisposition, insult, response and organ dysfunction (PIRO) score}

In a recent report, some investigators set out to formulate a prognostic scale for estimating mortality, which would be applicable to children with CAP. ${ }^{33}$ The study enrolled patients younger than 15 years with CAP who were hospitalized over a 10 -year period. A point-based scoring system based on the modified PIRO scale used in adults with pneumonia was applied to each hospitalized child, and it comprised the following variables: predisposition (age $<6$ months, comorbidity), insult (hypoxia [O2 saturation $<90 \%$ ], hypotension [according to age] and bacteremia), response (multilobar or complicated pneumonia) and organ dysfunction (kidney failure, liver failure and acute respiratory distress syndrome). One point was awarded for each criterion that was present (range: $0-10$ points). The association between the modified PIRO score and mortality was assessed by stratifying patients into four levels of risk: low ( $0-2$ points), moderate (3-4 points), high (5-6 points) and very high risk (7-10 points). The results showed a significant positive correlation between mortality rates and modified PIRO scores, causing the authors to conclude that the score accurately discriminated the probability of mortality in children hospitalized with CAP and thus could be a reliable tool to select patients for admission to ICU and for adjunctive therapy in clinical trials. ${ }^{33}$ Although this tool is the modified version of the score initially designed for CAP in adult patients, ${ }^{34}$ it appears to be the only prognostic scoring system that has been shown to be applicable in pediatric CAP.

\section{Prognostic indicators for complications of CAP}

Severe CAP is associated with serious complications, such as respiratory insufficiency, sepsis and mortality. Some studies have highlighted the prognostic indicators of these complications. ${ }^{35-39}$ In a prospective, randomized study, a group of investigators compared the following: standard treatment plus noninvasive positive pressure ventilation (NPPV) delivered through a face mask; and standard treatment alone in 
patients with severe CAP complicated by acute respiratory failure. ${ }^{35}$ Interestingly, the use of NPPV was found to be well tolerated, safe and associated with a significant reduction in both respiratory rate and the need for endotracheal intubation. Thus, it was concluded that in selected patients with acute respiratory failure, NPPV was associated with a significant reduction in the rate of endotracheal intubation and duration of ICU admission. ${ }^{35}$ On the other hand, other authors reported that routine clinical and biochemical information can be used to predict early respiratory failure in patients with severe CAP. ${ }^{36}$ For instance, respiratory failure was found to be independently associated with altered mental state, pulmonary arterial hypertension $(\mathrm{PaH})<7.35 \mathrm{mmHg}$ and $\mathrm{PaO}_{2}<60 \mathrm{mmHg}$. Furthermore, a history of heart failure was inversely associated with respiratory failure, while patients who failed to respond had a higher 28-day mortality rate and a longer period of hospitalization. ${ }^{36}$ In one study among adult patients with pneumococcal CAP, sepsis and the CRB-65 scores were used to allocate patients to subgroups with low, intermediate and high risk. ${ }^{37}$ The authors observed that even though both scores performed equally well in predicting mortality, the prediction of survival in the intermediate-risk group appeared more accurate with the sepsis score.

Notably, as CAP becomes more complicated with severe sepsis and septic shock, blood lactate concentration is increased as a result of anaerobic production (via the $\mathrm{Na}^{+}$ $\mathrm{K}^{+}$-ATPase channel) and a decrease in lactate utilization. ${ }^{38} \mathrm{In}$ another study among critically ill geriatric patients admitted into the ICU with severe CAP, lactate clearance was found to be a useful and inexpensive biomarker, as well as a reliable predictor of patient outcome. ${ }^{39}$ Thus, it is an important prognostic tool for CAP-related sepsis.

\section{Biomarkers in geriatric and pediatric CAP: the progress so far}

An ideal biomarker for CAP should lead to a prompt diagnosis, have a prognostic value and facilitate therapeutic decision-making. ${ }^{16}$ Biomarkers provide reliable information about host response to infection as well as pathogenic activity within the host, which can serve to support clinical parameters in making decisions. ${ }^{15}$ Hitherto, biomarkers have been used to differentiate bacterial and viral etiologies in childhood CAP. ${ }^{40,41}$ Recently, they have emerged as predictors of disease severity and prognosis. ${ }^{42-44}$

From a pathophysiological perspective, it is possible that the combination of several biomarkers reflecting different aspects of CAP (infection, cardiovascular impairment, sepsis and respiratory failure) can be useful. Hence, the novel biomarkers fall into these major groups: inflammatory markers, namely, procalcitonin (PCT), C-reactive protein (CRP) and white blood cell (WBC) count; cardiovascular markers, such as proadrenomedullin (proADM), proatrial natriuretic peptide (proANP), proarginine vasopressin (copeptin) and C-terminal proendothelin-1 (CT-proET-1); and biomarkers of stress, such as cortisol. ${ }^{45}$ Adrenomedullin (ADM) has several actions, including immune modulation, vasodilation, direct bactericidal activity and electrolyte homeostasis. Endothelin-1 is a potent vasoconstrictor, and its endothelial synthesis is triggered by hypoxia and pulmonary infections. ${ }^{46}$ PCT is a specific marker for bacterial infections, while arginine vasopressin plays a role in the regulation of cardiovascular and osmotic homeostasis. Plasma levels of ANP are elevated in patients with chronic heart failure and sepsis and predict impaired left ventricular ejection fraction. ${ }^{47,48}$

Notably, these biomarkers have not only been established

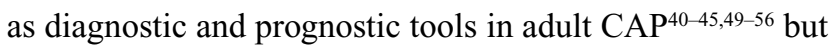
have also been demonstrated to have wide applicability in pediatric CAP. ${ }^{57-73}$

In one major prospective study that compared these new biomarkers in predicting short- and long-term all-cause mortality in adult CAP, the investigators found that the cardiovascular biomarkers (proANP, copeptin, CT-proET-1 and proADM) were not only good predictors of short- and long-term outcomes in CAP, but they were also superior to inflammatory markers, and at least comparable to the CRB-65 score, in terms of performance. ${ }^{45}$ ProADM was noted as the biomarker with the best performance, while the combination of CRB-65 score and proADM was suggested as the best predictor for both short- and long-term mortalities. ${ }^{45}$

Some recent studies that investigated the utility of serum biomarkers to measure CAP severity and predict prognosis have focused on CRP, PCT and cortisol ${ }^{49-52}$ For example, a study of 185 adult patients who had PCT measured within 24 hours of the diagnosis of CAP showed that PCT levels correlated with the PSI score and the development of complications. ${ }^{52}$ Levels were also elevated in nonsurvivors when compared with that in survivors. Serial measurements of PCT have similarly been used to define prognosis in severe CAP patients. ${ }^{49}$ Other investigators have reported that nonsurvivors had significantly higher PCT levels; with serial measurements, survivors had a reduction in PCT levels, while nonsurvivors had a rise by the third day. ${ }^{49}$

Interestingly, researchers are now beginning to combine the findings of biomarker estimations with the results of the prognostic scoring systems in adult patients with CAP. 
In one study that evaluated PCT, CRP and CRB-65 in 1,672 adult patients with the disease, PCT levels on admission were elevated in nonsurvivors, and the receiver operating characteristic (ROC) curve for survival was similar for PCT and CRB-65, but each was better than CRP. ${ }^{53}$ A reduced PCT level specifically predicted a low risk of mortality independent of CRB-65 category.

In another study of 278 adult patients presenting with CAP to an emergency department in Switzerland, the authors showed that cortisol levels could be used to predict severity of disease and mortality-related outcome. ${ }^{54}$ Free and total cortisol levels were found to be correlated with severity of CAP as shown by the PSI score; a level of total cortisol $>960$ $\mathrm{nmol} / \mathrm{L}$ had a sensitivity of $75 \%$ and a specificity of $71.7 \%$ for predicting mortality. In a related study, baseline cortisol was noted to be the best predictor of mortality among 72 adult patients with severe CAP who were admitted to the ICU, as mortality was more frequent in those with higher baseline levels. ${ }^{55}$ When baseline cortisol was used to predict mortality, it was more accurate than CURB-65 using the area under the ROC curve for mortality, but CRP was not a good biomarker for the same purpose. In one systematic review and meta-analysis, the authors nonetheless concluded that despite the fact that biomarkers are able to predict mortality with moderate-to-good accuracy in adult CAP, they have no clear advantage over CAP-specific scores. ${ }^{56}$ Nevertheless, in a recent review that aimed to determine the role of PCT in predicting CAP severity in children, there appears to be an increase in studies confirming its utility in CAP management in pediatric patients. ${ }^{61}$ In one of such recently published studies, 119 children aged 1-14 years were admitted for radiographically documented CAP, and baseline PCT, CRP and routine laboratory tests were conducted on admission. The investigators found that PCT was correlated to the main inflammatory markers in children with CAP; CRP, unlike PCT, was able to predict the severity of CAP, confirming its usefulness in the management of the disease. ${ }^{57}$

The medical literature is currently replete with other reports that indicate the utility of biomarkers as diagnostic, prognostic and discriminatory tools, as well as being a possible therapeutic guide in pediatric CAP. ${ }^{58-73}$ For instance, as a discriminatory tool, Esposito et $\mathrm{al}^{60}$ investigated the usefulness of biomarkers such as lipocalin-2 (LIP2) and syndecan 4 (SYN 4) in differentiating bacterial from viral etiology in CAP. They estimated the serum levels of these biomarkers together with the WBC counts and CRP levels in 110 children aged $<14$ years who were hospitalized for radiographically proven CAP. However, their findings showed that the estimation of LIP2 and SYN4 levels did not obviate the need for using clinical and laboratory parameters to define the etiology and severity of pediatric CAP. ${ }^{60}$

In a retrospective cohort study of 108 pediatric patients divided into four categories of those with bacterial pneumonia or bronchitis and those with nonbacterial pneumonia or bronchitis, Hoshina et $\mathrm{al}^{64}$ compared the WBC and neutrophil counts, as well as the CRP and PCT levels, among the cohorts. The authors were able to demonstrate that PCT was the most useful marker to differentiate bacterial from nonbacterial pneumonia, while neutrophil count was the major contributor to the discrimination of bacterial from nonbacterial bronchitis. ${ }^{64}$ In contrast, Don et al, ${ }^{69}$ in their study of 101 children with radiographically proven CAP, reported that WBC count, CRP, PCT and erythrocyte sedimentation rate (ESR), or their combinations, showed a limited discriminatory role during screening between bacterial and viral pediatric CAP. They further noted that if all or most of these markers were raised, bacterial etiology was most likely, but reduced values did not preclude bacterial etiology. Their findings were corroborated by another study, ${ }^{72}$ which observed that CRP, PCT, WBC and ESR had only limited value in differentiating bacterial from viral pneumonia; none of the combinations of these markers showed sufficient sensitivity and specificity to be used in pediatric CAP. Thus, the evidence is still weak - and there is no consensus yet - on the utility of biomarkers in differentiating the microbial etiology of CAP. In addition, Tumgor et $\mathrm{al}^{70}$ similarly reported that interleukin (IL)-6, IL-8 and CRP levels were elevated in children with CAP and HAP but were not useful in differentiating the various etiologic agents. Remarkably, one published report from a prospective study of 310 children admitted for uncomplicated CAP used a PCT cutoff value to guide antibiotic therapy in these patients and showed that an algorithm-based approach can significantly reduce antibiotic use and antibiotic-related adverse reactions in those with uncomplicated disease. ${ }^{67}$ However, the authors could not validate the risk of using this approach in severe cases of CAP because the study included mainly children with mild-to-moderate forms of the disease.

Again, several reports show the diagnostic utility of established and emerging biomarkers in pediatric CAP. ${ }^{58,61,62,65,66,68,71,73}$ In a recently published study, Li et al ${ }^{58}$ used proteomics to identify 27 potential plasma proteins in children with $M$. pneumoniae pneumonia (MPP) and found that human apolipoprotein C-I precursor (APOC1) was a potential novel biomarker for the rapid and noninvasive 
diagnosis of MPP in children, an observation that the authors believed could offer new insights into the pathogenesis and biomarker selection of MPP in pediatric patients.

Alcoba et $\mathrm{l}^{62}$ evaluated the predictive value of proADM and copeptin for complicated CAP (evidenced by bacteremia and empyema) in 88 pediatric patients aged $0-16$ years who were seen in an emergency setting. The investigators noted that only proADM appeared to be a reliable predictor for complicated CAP and could thus aid the clinician with decisions such as in-patient care and choice of the route of antibiotic administration. Similarly, other researchers have reported the diagnostic and prognostic utility of this biomarker ${ }^{66}$ In a prospective observational study of children with CAP seen at the emergency department, proADM values were analyzed in relation to clinical parameters such as need for oxygen therapy, duration of oxygen therapy, disease complications, pyrexia, antibiotic therapy, admission to ICU and duration of hospitalization; specifically children who had complications such as pleural effusion had higher levels of proADM. ${ }^{66}$

In another study by Wrotek et al, ${ }^{65}$ soluble urokinase plasminogen activator receptor (suPAR) level was estimated in 227 children with CAP and in 119 healthy counterparts. The marker, which is an activator of the immune system, was significantly more elevated in children with CAP than in healthy subjects. In addition, suPAR level was significantly higher in children with severe form of the disease compared with those who had nonsevere illness, indicating that its elevation may also reflect the severity of pneumonia. The same authors also demonstrated a positive correlation between serum suPAR and nonspecific inflammatory markers, such as $\mathrm{CRP}$ and PCT, in pediatric CAP. ${ }^{63}$

Elsewhere, in South Korea, a group of researchers reported that serum PCT levels had better diagnostic accuracy for lobar pneumonia compared with the predictive ability of serum CRP level and ESR. ${ }^{68}$ They also noted a significant correlation of the PCT level with the CRP level, ESR and WBC count, suggesting that serum PCT level was a better marker than CPR and ESR for the diagnosis of lobar pneumonia in pediatric CAP. ${ }^{68}$ In a Chinese study that investigated the clinical value of the expression of neutrophil surface cluster of differentiation (CD)64 in the diagnosis of CAP in children, the authors observed that the CD64 index and CRP levels were significantly higher in patients with bacterial pneumonia than in their counterparts with viral and mycoplasma pneumonia. ${ }^{73}$ In addition, the CD64 index was observed to be significantly higher in children with severe pneumonia than in those with milder forms of the disease, while its expression also significantly decreased in those with severe bacterial infection after antibiotic therapy, indicating that its determination contributes to early diagnosis of bacterial CAP. ${ }^{73}$ Finally, some biomarkers have also been documented as prognostic tools in pediatric CAP in a recent study in India, which investigated the possible role of systemic immune and inflammatory mediators as biomarkers that could predict CAP severity or outcome. ${ }^{59}$ The researchers observed that IL-6, IL-8, IL-13, interferon-gamma (IFN- $\gamma$ ) and lower $\mathrm{C}-\mathrm{C}$ motif chemokine ligand 22 (CCL22) levels were significantly more elevated in patients with severe CAP compared with those with mild CAP. Furthermore, based on higher macrophage inflammatory protein-1 alpha (MIP-1 $\alpha$ ), IL-8, IL-17 or lower CCL22-response pattern at the time of enlistment, children with fatal outcome displayed a markedly different pattern of inflammatory response compared with those grouped with the same disease severity, but with nonlife-threatening outcome. ${ }^{59}$

\section{Conclusion}

Most of the prognostic scoring systems currently under use are more applicable to CAP in adult patients. So far, only the modified PIRO score has been applied to pediatric patients. The most evaluated tools - PSI and CURB-65 - are accurate for predicting mortality in adults with CAP but are not direct indicators of disease severity.

Thus, they cannot replace the use of clinical assessment to determine hospital admission or ICU care. At best, these scoring systems are decision support tools that cannot be utilized as the gold standard for site-of-care decisions. ${ }^{18}$

Since none of the tools is without its limitations, there has been a paradigm shift to incorporate biomarkers in risk stratification because they are good predictors of disease severity and outcome, irrespective of age group. Although the role of biomarkers was initially more established in geriatric CAP, they have now been proven to be useful in the diagnosis of pediatric CAP, as well as in predicting severity and outcome in children. Despite the progress made so far in applying these biomarkers in pediatric CAP, more research is still needed to validate their diagnostic and prognostic utility in the disease.

\section{Acknowledgment}

The authors acknowledge the invaluable information obtained from the article by Niederman. ${ }^{18}$

\section{Disclosure}

The authors report no conflicts of interest in this work. 


\section{References}

1. Bradley JS, Byington CL, Shah SS, et al; Pediatric Infectious Diseases Society and the Infectious Diseases Society of America. The management of community-acquired pneumonia in infants and children older than 3 months of age: clinical practice guidelines by the Pediatric Infectious Diseases Society and the Infectious Diseases Society of America. Clin Infect Dis. 2011;53(7):e25.

2. Ewig S. Community-acquired pneumonia: definition, epidemiology, and outcome. Semin Respir Infect. 1999;14(2):94-102.

3. British Thoracic Society Standards of Care Committee. British Thoracic Society guidelines for the management of community acquired pneumonia in childhood. Thorax. 2002;57(suppl 1):i1-i24.

4. Murphy TF, Henderson FW, Clyde WA Jr, Collier AM, Denny FW. Pneumonia: an eleven-year study in a pediatric practice. Am J Epidemiol. 1981;113(1):12-21.

5. Jokinen C, Heiskanen L, Juvonen H, et al. Incidence of communityacquired pneumonia in the population of four municipalities in Eastern Finland. Am J Epidemiol. 1993;137(9):977-988.

6. Redd SC, Vreuls R, Metsing M, Mohobane PH, Patrick E, Moteetee M. Clinical signs of pneumonia in children attending a hospital outpatient department in Lesotho. Bull World Health Organ. 1994;72(1):113-118.

7. McIntosh K. Community-acquired pneumonia in children. $N$ Engl J Med. 2002;346:429-437.

8. Principi N, Esposito S. Mycoplasma pneumoniae and Chlamydia pneumoniae cause lower respiratory tract disease in pediatric patients. Curr Opin Infect Dis. 2002;15:295-300.

9. Heiskanen-Kosma T, Korppi M, Jokinen C, et al. Etiology of childhood pneumonia: serologic results of a prospective, population-based study. Pediatr Infect Dis J. 1998;17(11):986-991.

10. von Baum H, Ewig S, Marre R, et al; Competence Network for Community Acquired Pneumonia Study Group. Community-acquired Legionella pneumonia: new insights from the German Competence Network for community acquired pneumonia. Clin Infect Dis. 2008;46(9):1356-1364.

11. van de Garde EM, Endeman H, van Hemert RN, et al. Prior outpatient antibiotic use as predictor for microbial aetiology of communityacquired pneumonia: hospital-based study. Eur J Clin Pharmacol. 2008;64(4):405-410.

12. Huang HH, Zhang YY, Xiu QY, et al. Community-acquired pneumonia in Shanghai, China: microbial etiology and implications for empirical therapy in a prospective study of 389 patients. Eur J Clin Microbiol Infect Dis. 2006;25(6):369-374.

13. Peto L, Nadjm B, Horby P, et al. The bacterial aetiology of adult community-acquired pneumonia in Asia: a systematic review. Trans $R$ Soc Trop Med Hyg. 2014;108(6):326-337.

14. Grossman RF. Community-Acquired Pneumonia: Advances in Management. ACCP Pulmonary Medicine Board Review. 26th ed. Glenview, IL: ACCP; 2009.

15. Florin TA, Ambroggio L. Biomarkers for community-acquired pneumonia in the emergency department. Curr Infect Dis Rep. 2014;16(12):451.

16. Krüger S, Welte T. Biomarkers in community-acquired pneumonia. Expert Rev Respir Med. 2012;6(2):203-214.

17. Kolditz M, Ewig S, Höffken G. Management-based risk prediction in community-acquired pneumonia by scores and biomarkers. Eur Respir J. 2013;41(4):974-984.

18. Niederman MS. Making sense of scoring systems in community acquired pneumonia. Respirology. 2009;14(3):327-335.

19. Fine MJ, Auble TE, Yealy DM, et al. A prediction rule to identify lowrisk patients with community- acquired pneumonia. $N$ Engl J Med. 1997;336(4):243-250.

20. Rello J, Rodriguez A. Severity of illness assessment for managing community acquired pneumonia. Intensive Care Med. 2007;33(12): 2043-2044.

21. Lim WS, van der Eerden MM, Laing R, et al. Defining community acquired pneumonia severity on presentation to hospital: an international derivation and validation study. Thorax. 2003;58(5):377-382.
22. Capelastegui A, España PP, Quintana JM, et al. Validation of a predictive rule for the management of community-acquired pneumonia. Eur Respir J. 2006;27:151-157.

23. Bauer TT, Ewig S, Marre R, Suttorp N, Welte T; the CAPNETZ Group. CRB-65 predicts death from community-acquired pneumonia. J Intern Med. 2006;260(1):93-101.

24. Niederman MS, Feldman C, Richards GA. Combining information from prognostic scoring tools for CAP: an American view on how to get the best of both worlds. Eur Respir J. 2006;27:9-11.

25. Aujesky D, Auble TE, Yealy DM, et al. Prospective comparison of three validated prediction rules for prognosis in community-acquired pneumonia. Am J Med. 2005;118(4):384-392.

26. Ewig S, De Roux A, Bauer T, et al. Validation of predictive rules and indices of severity for community acquired pneumonia. Thorax. 2004; 59(5):421-427.

27. Niederman MS, Mandell LA, Anzueto A, et al; American Thoracic Society. Guidelines for the management of adults with communityacquired pneumonia. Diagnosis, assessment of severity, antimicrobial therapy, and prevention. Am J Respir Crit Care Med. 2001;163: 1730-1754.

28. Mandell LA, Wunderink RG, Anzueto A, et al. Infectious Diseases Society of America/American Thoracic Society consensus guidelines on the management of community-acquired pneumonia in adults. Clin Infect Dis. 2007;44(suppl 2):S27-S72.

29. España PP, Capelastegui A, Gorordo I, et al. Development and validation of a clinical prediction rule for severe community-acquired pneumonia. Am J Respir Crit Care Med. 2006;174(11):1249-1256.

30. Charles PC, Wolfe R, Whitby M, et al; Australian CommunityAcquired Pneumonia Study Collaboration. SMART-COP: a tool for predicting the need for intensive respiratory or vasopressor support in community-acquired pneumonia. Clin Infect Dis. 2008;47(3): 375-384.

31. Chalmers JD, Singanayagam A, Hill AT. Predicting the need for mechanical ventilation and/or inotropic support for young adults admitted to the hospital with community-acquired pneumonia. Clin Infect Dis. 2008;47(12):1571-1574.

32. British Thoracic Society; Myint PK, Kamath AV, Vowler SL, Maisey DN, Harrison BD. Severity assessment criteria recommended by the British Thoracic Society (BTS) for community-acquired pneumonia (CAP) and older patients. Should SOAR (systolic blood pressure, oxygenation, age and respiratory rate) criteria be used in older people? A compilation study of two prospective cohorts. Age Ageing. 2006;35(3): 286-291.

33. Araya S, Lovera D, Zarate C, et al. Application of a Prognostic Scale to estimate the mortality of children hospitalized with community-acquired pneumonia. Pediatr Infect Dis J. 2016;35(4):369-373.

34. Rello J, Rodriguez A, Lisboa T, Gallego M, Lujan M, Wunderink R. PIRO score for community-acquired pneumonia: a new prediction rule for assessment of severity in intensive care unit patients with community-acquired pneumonia. Crit Care Med. 2009;37(2):456-462.

35. Confalonieri M, Potena A, Carbone G, Della Porta R, Tolley EA, Meduri GU. Acute respiratory failure in patients with severe communityacquired pneumonia: a prospective randomized evaluation of noninvasive ventilation. Am J Resp Crit Care Med. 1999;160(5):1585-1591.

36. Hoogewerf M, Oosterheert JJ, Hak E, Hoepelman IM, Bonten MJM. Prognostic factors for early clinical failure in patients with severe community-acquired pneumonia. Clin Microbiol Infect. 2006;12:1097-1104.

37. Schaaf B, Kruse J, Rupp J, et al. Sepsis severity predicts outcome in community-acquired pneumococcal pneumonia. Eur Respir J. 2007;30(3):517-524.

38. James JH, Luchette FA, McCarter FD, Fischer JE. Lactate is an unreliable indicator of tissue hypoxia in injury or sepsis. Lancet. 1999; 354(9177):505-508.

39. Abd Elaziz Mohamed K, Abd Elgalil Ahmed D. Prognostic value of lactate clearance in severe community acquired pneumonia. Egypt $J$ Chest Dis Tuberculosis. 2014;63(4):1053-1058. 
40. Moulin F, Raymond J, Lorrot M, et al. Procalcitonin in children admitted to hospital with community acquired pneumonia. Arch Dis Child. 2001;84(4):332-336.

41. Toikka P, Irjala K, Juvén T, et al. Serum procalcitonin, C-reactive protein and interleukin-6 for distinguishing bacterial and viral pneumonia in children. Pediatr Infect Dis J. 2000;19(7):598-602.

42. Krüger S, Ewig S, Kunde J, Hartmann O, Suttorp N, Welte T. Pro-atrial natriuretic peptide and pro-vasopressin to predict short- and long-term survival in community-acquired pneumonia. Thorax. 2010;65:208-214.

43. Krüger S, Ewig S, Marre R, et al. Procalcitonin predicts patients at low risk of death from community-acquired pneumonia. Eur Respir J. 2008;31:349-355.

44. Koivula I, Sten M, Makela P. Prognosis after community-acquired pneumonia in the elderly: a population-based 12-year follow-up study. Arch Intern Med. 1999;159:1550-1555.

45. Krüger S, Ewig S, Giersdorf S, Hartmann O, Suttorp N, Welte T. Cardiovascular and inflammatory biomarkers to predict short- and long-term survival in community-acquired pneumonia. Am J Respir Crit Care Med. 2010;182(11):1426-1434.

46. Carpenter TC, Schornberg S, Stenmark KR. Endothelin-mediated increases in lung VEGF content promote vascular leak in young rats exposed to viral infection and hypoxia. Am J Physiol Lung Cell Mol Physiol. 2005;289:L1075-L1082.

47. Morgenthaler NG, Struck J, Christ-Crain M, Bergmann A, Müller B. Pro-atrial natriuretic peptide is a prognostic marker in sepsis, similar to the APACHE II score: an observational study. Crit Care. 2003; 9(1):R37-R45

48. Jochberger S, Morgenthaler NG, Mayr VD, et al. Copeptin and arginine vasopressin concentrations in critically ill patients. J Clin Endocrinol Metab. 2006;91(11):4381-4386.

49. Boussekey N, Leroy O, Alfandari S, Devos P, Georges H, Guery B. Procalcitonin kinetics in the prognosis of severe community-acquired pneumonia. Intensive Care Med. 2006;32:469-472.

50. Boussekey N, Leroy O, Georges H, Devos P, D’Escrivan T, Guery B. Diagnostic and prognostic values of admission procalcitonin levels in community-acquired pneumonia in an intensive care unit. Infection. 2005;33(4):257-263.

51. Christ-Crain M, Stolz D, Bingisser R, et al. Procalcitonin guidance of antibiotic therapy in community-acquired pneumonia: a randomized trial. Am J Respir Crit Care Med. 2006;174(1):84-93.

52. Masia M, Gutierrez F, Shum C, et al. Usefulness of procalcitonin levels in community-acquired pneumonia according to severity index. Chest. 2005;128:2223-2229.

53. Huang DT, Weissfeld LA, Kellum JA, et al; GenIMS Investigators. Risk prediction with procalcitonin and clinical rules in community-acquired pneumonia. Ann Emerg Med. 2008;52:48-58.

54. Christ-Crain M, Stolz D, Jutla S, et al. Free and total cortisol levels as predictors of severity and outcome in community-acquired pneumonia. Am J Respir Crit Care Med. 2007;176(9):913-920.

55. Salluh JIF, Bozza FA, Soares M, et al. Adrenal response in severe community-acquired pneumonia: impact on outcomes and disease severity. Chest. 2008;134:947-954.

56. Viasus D, Del Rio-Pertuz G, Simonetti AF, et al. Biomarkers for predicting short-term mortality in community-acquired pneumonia: a systematic review and meta-analysis. J Infect. 2016;72(3):273-282.
57. Agnello L, Bellia C, Di Gangi M, et al. Utility of serum procalcitonin and C-reactive protein in severity assessment of community-acquired pneumonia in children. Clin Biochem. 2016;49(1-2):47-50.

58. Li J, Sun L, Xu F, et al. Screening and identification of APOC1 as a novel potential biomarker for differentiate of Mycoplasma pneumoniae in children. Front Microbiol. 2016;7:1961.

59. Saghafian-Hedengren S, Mathew JL, Hagel E, et al. Assessment of cytokine and chemokine signatures as potential biomarkers of childhood community-acquired pneumonia severity: a nested cohort study in India. Pediatr Infect Dis J. 2017;36(1):102-108.

60. Esposito S, Bianchini S, Gambino M, et al. Measurement of lipocalin-2 and syndecan-4 levels to differentiate bacterial from viral infection in children with community-acquired pneumonia. BMC Pulm Med. 2016;16(1):103.

61. Giulia B, Luisa A, Concetta S, Bruna LS, Chiara B, Marcello C. Procalcitonin and community-acquired pneumonia (CAP) in children. Clin Chim Acta. 2015;451(pt B):215-218.

62. Alcoba G, Manzano S, Lacroix L, Galetto-Lacour A, Gervaix A. Proadrenomedullin and copeptin in pediatric pneumonia: a prospective diagnostic accuracy study. BMC Infect Dis. 2015;15:347.

63. Wrotek A, Jackowska T, Pawlik K. Soluble urokinase plasminogen activator receptor: an indicator of pneumonia severity in children. $A d v$ Exp Med Biol. 2015;835:1-7.

64. Hoshina T, Nanishi E, Kanno S, Nishio H, Kusuhara K, Hara T. The utility of biomarkers in differentiating bacterial from non-bacterial lower respiratory tract infection in hospitalized children: difference of the diagnostic performance between acute pneumonia and bronchitis. J Infect Chemother. 2014;20(10):616-620.

65. Wrotek A, Pawlik K, Jackowska T. Soluble receptor for urokinase plasminogen activator in community-acquired pneumonia in children Adv Exp Med Biol. 2013;788:329-334.

66. Sardà Sánchez M, Hernández JC, Hernández-Bou S, Teruel GC, Rodríguez JV, Cubells CL. Pro-adrenomedullin usefulness in the management of children with community-acquired pneumonia, a preliminar(y) prospective observational study. BMC Res Notes. 2012;5:363.

67. Esposito S, Tagliabue C, Picciolli I, et al. Procalcitonin measurements for guiding antibiotic treatment in pediatric pneumonia. Respir Med. 2011;105(12):1939-1945.

68. Lee JY, Hwang SJ, Shim JW, et al. Clinical significance of serum procalcitonin in patients with community-acquired lobar pneumonia. Korean J Lab Med. 2010;30(4):406-413.

69. Don M, Valent F, Korppi M, Canciani M. Differentiation of bacterial and viral community-acquired pneumonia in children. Pediatr Int. 2009;51(1):91-96.

70. Tumgor G, Celik U, Alabaz D, et al. Aetiological agents, interleukin-6, interleukin-8 and CRP concentrations in children with community- and hospital-acquired pneumonia. Ann Trop Paediatr. 2006;26(4):285-291.

71. Ballin A, Osadchy A, Klivitsky A, Dalal I, Lishner M. Age-related leukocyte and cytokine patterns in community-acquired bronchopneumonia. Isr Med Assoc J. 2006;8(6):388-390.

72. Korppi M. Non-specific host response markers in the differentiation between pneumococcal and viral pneumonia: what is the most accurate combination? Pediatr Int. 2004;46(5):545-550.

73. Cai Q, Xu MY. [Value of neutrophil CD64 in the diagnosis of community acquired pneumonia in children]. [Article in Chinese]. Zhongguo Dang Dai Er Ke Za Zhi. 2012;14(11):819-822. 


\section{Publish your work in this journal}

Pediatric Health, Medicine and Therapeutics is an international, peerreviewed, open access journal publishing original research, reports, editorials, reviews and commentaries. All aspects of health maintenance, preventative measures and disease treatment interventions are addressed within the journal. Practitioners from all disciplines are invited to submit their work as well as healthcare researchers and patient support groups. The manuscript management system is completely online and includes a very quick and fair peer-review system. Visit http://www.dovepress.com/ testimonials.php to read real quotes from published authors.

Submit your manuscript here: http://www.dovepress.com/pediatric-health-medicine-and-therapeutics-journal 